\title{
Electro-optical testing of fully depleted CCD image sensors for the Large Synoptic Survey Telescope camera
}

\section{Citation}

Doherty, Peter E., et al. 2014. Electro-optical testing of fully depleted CCD image sensors for the Large Synoptic Survey Telescope camera. In the Proceedings of High Energy, Optical, and Infrared Detectors for Astronomy VI (SPIE 9154), June 22, 2014, Montreal, Quebec, Canada: August 12, 2014. doi:10.1117/12.2056733

\section{Published Version}

doi:10.1117/12.2056733

\section{Permanent link}

http://nrs.harvard.edu/urn-3:HUL.InstRepos:20562432

\section{Terms of Use}

This article was downloaded from Harvard University's DASH repository, and is made available under the terms and conditions applicable to Other Posted Material, as set forth at http:// nrs.harvard.edu/urn-3:HUL.InstRepos:dash.current.terms-of-use\#LAA

\section{Share Your Story}

The Harvard community has made this article openly available.

Please share how this access benefits you. Submit a story.

Accessibility 


\title{
Electro-Optical Testing of Fully Depleted CCD Image Sensors for the Large Synoptic Survey Telescope Camera
}

\author{
Peter E. Doherty ${ }^{\mathrm{a}}$, Pierre Antilogus ${ }^{\mathrm{b}}$, Pierre Astier ${ }^{\mathrm{b}}$, James Chiang ${ }^{\mathrm{c}}$, D. Kirk Gilmore ${ }^{\mathrm{c}}$, Augustin \\ Guyonnet $^{\mathrm{b}}$, Dajun Huang ${ }^{\mathrm{d}}$, Heather Kelly ${ }^{\mathrm{c}}$, Ivan Kotov ${ }^{\mathrm{d}}$, Petr Kubanek ${ }^{\mathrm{e}}$, Andrei Nomerotski ${ }^{\mathrm{d}}$, Paul \\ O’Connor $^{\mathrm{d}}$, Andrew Rasmussen ${ }^{\mathrm{c}}$, Vincet J. Riot ${ }^{\mathrm{f}}$, Christopher W. Stubbs ${ }^{\mathrm{a}}$, Peter Takacs ${ }^{\mathrm{d}}, \mathrm{J}$. \\ Anthony Tyson ${ }^{\mathrm{g}}$, Kurt Vetter ${ }^{\mathrm{d}}$ \\ aDepartment of Physics, Harvard University, Cambridge, MA, USA; \\ bInstitut National de Physique Nucléaire et de Physique des Particules (IN2P3), 3 rue Michel- \\ Ange, Paris Cedex 16, France \\ cSLAC National Accelerator Laboratory, 2575 Sand Hill Rd., Menlo Park, CA USA \\ dBrookhaven National Laboratory, Upton, NY, USA; \\ eInstitute of Physics, Academy of Sciences, Praha, Czech Republic ; \\ ${ }^{f}$ Lawrence Livermore National Laboratory, 7000 East Ave., Livermore, CA, USA \\ gDepartment of Physics, University of California, Davis, CA,USA
}

\begin{abstract}
The LSST Camera science sensor array will incorporate 189 large format Charge Coupled Device (CCD) image sensors. Each CCD will include over 16 million pixels and will be divided into 16 equally sized segments and each segment will be read through a separate output amplifier.

The science goals of the project require CCD sensors with state of the art performance in many aspects. The broad survey wavelength coverage requires fully depleted, 100 micrometer thick, high resistivity, bulk silicon as the imager substrate. Image quality requirements place strict limits on the image degradation that may be caused by sensor effects: optical, electronic, and mechanical.

In this paper we discuss the design of the prototype sensors, the hardware and software that has been used to perform electro-optic testing of the sensors, and a selection of the results of the testing to date. The architectural features that lead to internal electrostatic fields, the various effects on charge collection and transport that are caused by them, including charge diffusion and redistribution, effects on delivered PSF, and potential impacts on delivered science data quality are addressed.
\end{abstract}

\section{INTRODUCTION}

The Large Synoptic Survey Telescope (LSST) will combine an 8.4 meter telescope with a light-gathering power among the largest in the world and a camera made up of more than 3 billion pixels. Data on billions of galaxies will be used to create a three dimensional map of the Universe with unprecedented depth and detail. This map will then be used to tomographically map dark matter, chart the clustering of galaxies, and to characterize the properties of dark energy. In addition, a wide range of other topics in astrophysics will be probed using the 15 Terabytes of imaging data that the system will produce each night.

Of particular importance in executing the LSST science mission is the fidelity of both the photometric and astrometric measurements that can be made using the data that the instrument produces. As example, the detection and distribution mapping of dark matter via the analysis of subtle geometric distortion of galactic shapes caused by gravitational lensing ("weak lensing") requires precision in both photometric and astrometric measurement.

The science goals of the project ${ }^{1}$ require CCD sensors with state of the art performance in many aspects. The telescope plate scale and expected image quality require a sensor pixel size of no more than 10 micrometers square. The broad survey wavelength coverage and sensitivity requirements at long wavelengths $(\sim 1000 \mathrm{~nm})$ dictate the use of thick, fully depleted, high resistivity bulk silicon as the imager substrate. Data throughput requirements impose a cadence of 15second exposures and two-second imager read times. Survey optical depth goals require a per-pixel amplifier read noise

High Energy, Optical, and Infrared Detectors for Astronomy VI, edited by Andrew D. Holland, James Beletic, Proc. of SPIE Vol. 9154, 915418 - @ 2014 SPIE · CCC code: 0277-786X/14/\$18 - doi: 10.1117/12.2056733 
of no more than 8 electrons rms. The two-second readout rate and relatively low readout noise requirements can only be met simultaneously by a highly segmented architecture. Image quality requirements place strict limits on the image degradation that may be caused by sensor effects: optical, electronic, and mechanical.

\section{PROTOTYPE LSST CCDS AND TEST FACILITIES}

\subsection{Baseline CCD architecture}

In order to meet the various requirements outlined above, a nominal sensor architecture and design was established. The features and specifications of the baseline design are shown in Figure 1.

\begin{tabular}{|c|c|c|c|}
\hline Requirement & Description & Value & Units \\
\hline \multirow[t]{4}{*}{ Architecture } & CCD Type & n-channel, full frame & \\
\hline & Pixel Size & $10 \times 10$ & microns \\
\hline & Segmentation & 16 & segments \\
\hline & Contiguity & continuous & \\
\hline \multirow[t]{11}{*}{ Electrical } & Frame Read Time & 2 & seconds \\
\hline & Read Noise & 8 (goal:5) & $e-r m s$ \\
\hline & Blooming Full Well & 175000 & e- maximum \\
\hline & Nonlinearity & \pm 2 & $\%, 1000$ to $90000 \mathrm{e}-$ \\
\hline & Serial CTE & 0.999995 & (@) 1000 and 50000 e- \\
\hline & Parallel CTE & 0.999998 & @ 1000 and 50000 e- \\
\hline & Active Area and Cosmetics & 99.5 & $\%$ of 16.129 Mpixels \\
\hline & Crosstalk & 0.19 (goal:0.02) & $\%$ \\
\hline & Dark Current, 95th Percentile & 0.2 & e-/pixel/second at $-95^{\circ} \mathrm{C}$ \\
\hline & Output Drive Capability & 10 & $\mathrm{pF}$ \\
\hline & Source Follower Power & 17 & $\mathrm{~mW}$ \\
\hline \multirow[t]{8}{*}{ Optical } & u-band Quantum Efficiency & 41 & $\%$ \\
\hline & g-band Quantum Efficiency & 78 & $\%$ \\
\hline & r-band Quantum Efficiency & 83 & $\%$ \\
\hline & i-band Quantum Efficiency & 82 & $\%$ \\
\hline & z-band Quantum Efficiency & 75 & $\%$ \\
\hline & y-band Quantum Efficiency & 21 & $\%$ measured at $-95^{\circ} \mathrm{C}$ \\
\hline & Pixel Response Non-uniformity & 5 & $\%$ rms \\
\hline & Point Spread Function & 5 & $\mu \mathrm{m} r \mathrm{rms}$ \\
\hline
\end{tabular}

Figure 1: LSST CCD architectural and electro-optical specifications

The LSST project has worked with two vendors to produce candidate devices for testing in LSST laboratories. Those devices have been tested and work on their detailed characterization continues.

\subsection{Sensor test facilities}

LSST has established several CCD test facilities. Brookhaven National Laboratory (BNL) is the lead institution for the development of the focal plane components and responsible for delivery of the sensors to the project. BNL has established a detector research laboratory and is in the process of constructing a production facility for the testing and integration of the final LSST focal plane components. Researchers at Harvard University have modified their CCD test facility for use on the LSST program and a specialized image sensor test laboratory has been constructed at the University of California at Davis. An additional test facility for both research and production work is under construction at the Institut National de Physique Nucléaire et de Physique des Particules (IN2P3) in Paris, France. Each of the LSST test laboratories is capable of performing complete electro-optical testing of the sensors against LSST specifications. In addition, specialized equipment at some laboratories allows for the study of sensor performance parameters in detail.

In the prototype testing phase of the project a set of automated laboratory control and image data collection facilities have been employed and/or developed in order to increase the testing throughput. LSST testing 
laboratories at BNL and Harvard have benefitted from the use of a customized version of the RTS2 observatory automation software ${ }^{3}$. This has simplified the automated control of the optical test benches and collection of the many image files on which much of our testing and characterization work depends. Higher-level programs written in Python allow for collection of complete electro-optic test data sets with minimal human intervention.

During the production phase of the program, further automation, with acquisition software based on the LSST Camera Control System (CCS) will allow for both automated data acquisition and archiving. The use of the CCS software will allow for the early integration of camera hardware and software components. In a similar vein, sensor electro-optical test data are currently reduced via a suite of software tools that is based on the developing LSST Data Management (DM) software framework, allowing researchers to begin to use the DM software as early in the project as is possible.

\section{SELECTED SENSOR CHARACTERISTICS}

In addition to testing of prototype sensors against established LSST sensor specifications, the test facilities have engaged in characterization of a number of CCD features that are not captured in those specifications. In this section we describe a few of those features and the tests that have been performed to study them.

\subsection{Image persistence}

LSST exposures will include stellar images that exceed (in some cases far exceed) the saturation point of the CCD imagers. This is unavoidable. Photo-generated charge from bright objects that exceed the detector pixel well capacity will spill into adjacent pixels in the column. This is known as 'blooming' and is common to all CCD detectors that do not incorporate 'anti-blooming' structures. That this excess charge will corrupt the information in pixels adjacent to those exposed to a high photon flux is accepted.

Under certain operating conditions, charge that should be contained in the buried channel of the CCD or 'spill over' into adjacent pixels can reach the $\mathrm{Si}-\mathrm{SiO} 2$ interface at the 'surface' of the CCD. This surface is the interface between the bulk silicon that contains the buried channel and the oxide layers that comprise the clocking gates. It has long been established that this interface is the site of defects in which electrons may be temporarily 'trapped'. Electrons trapped in these locations may either recombine with holes or be released subsequently. These released electrons, should they be released during a subsequent exposure and readout cycle, will appear in the subsequent image as a 'ghost' or residual image of the object from the prior exposure. The Poisson noise associated with the trapped signal that is included in the subsequent frame will contribute to the total readout noise of those pixels in those frames.

The operating condition that has the greatest potential to affect this 'trap and release' process is the upper rail of the image array (or 'parallel') clock signals. As part of LSST prototype sensor testing, we have studied the trapping of charge as a function of parallel clock voltages. The LSST cadence, pairs of 15-second exposures and 2-second readout periods at each pointing, is particularly sensitive to this effect as there is little time for trapped charge to be released prior to the next exposure and readout.

To study the effect, we have created an exposure sequence that partially simulates the LSST readout sequence. The sequence consists of two 15-second exposures and readout cycles back to back (with no clearing of the CCD prior to exposure) followed by a series of 15 -second dark integrations, simulating the follow-on exposures, but with no photon flux. This allows us to measure the amount of charge in the subsequent frames due to the prior exposures. In the real LSST cadence, it is likely that some clearing will be performed between exposure pairs. Given that, the cadence for this test, with no such clearing, represents a 'worst case' scenario.

In order to simulate a stellar (or slightly extended) source, the imaging hardware consists of a rear-illuminated pinhole that is imaged onto the CCD via a relay lens. Images were acquired in which the bright spot had a maximum signal equal to roughly $175 \mathrm{ke}$ - per pixel approximating the expected non-saturated full-well capacity of the device.

Figure 2 shows a section of the first dark integration following the two exposures for several settings of the parallel clock upper rail voltage. There is significant visual evidence for residual image in the frames representing parallel clock rail voltage of 10 Volts or more. As the charge packet is transferred towards the output node, interaction with the surface can continue. This is why, in the images below, the residual image is seen not just in the area that was subject to the illumination but also in the rows through which the charge was transferred. This effect is largely dependent on the parallel clocking voltages, as one would expect. It greatly increases the number of pixels affected and therefore the 
significance of the problem. Figure 3 displays the amount of residual signal found in the location of the imaged spot as a function of the voltage used as the upper rail of the parallel clock signals.
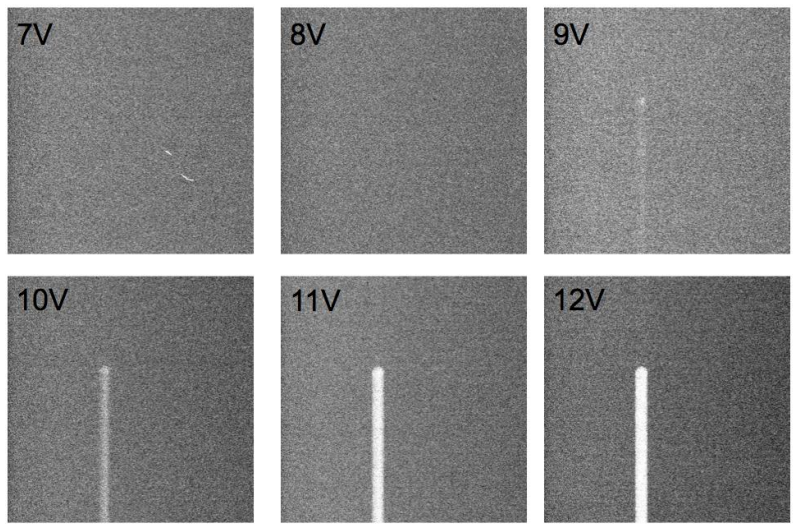

Figure 2: Residual Image after exposure to a flux of 175k e-/pixel

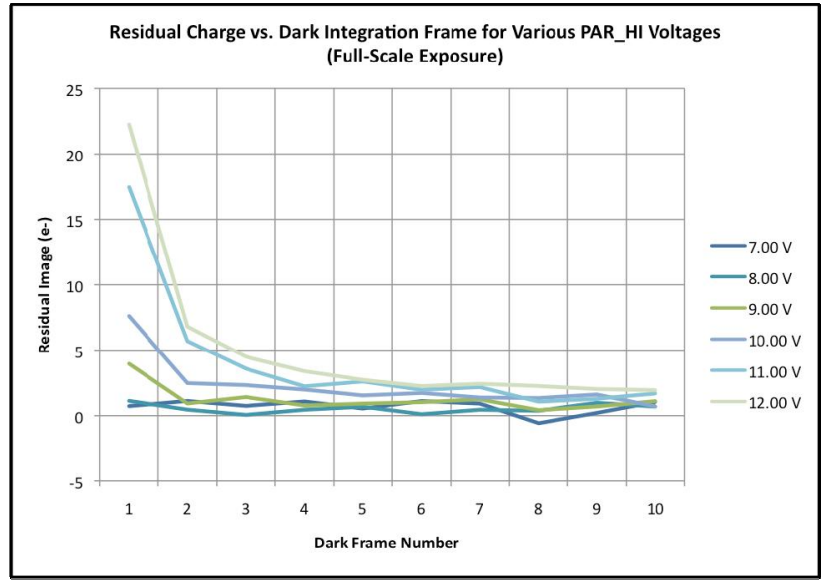

Figure 3: Residual Image as a function of frame number after exposure to a flux of $175 \mathrm{k} \mathrm{e}-/ \mathrm{pixel}$

At the $12 \mathrm{~V}$ setting the shot noise from the residual image is roughly equal to the read noise of the CCD, making it a significant contributor to the overall system noise. At settings of $10 \mathrm{~V}$ and below, the residual image is sufficiently low as to be effectively masked by sky noise in LSST pass-bands. Fortunately, sufficient full well capacity and charge transfer efficiency can be obtained with parallel clock voltages in the 9 to 10 volt range. In this range the residual image effect is non-zero but the additional noise that it superimposes on the subsequent frames is not a dominant term in the total noise.

During LSST prototype sensor testing, image persistence has only been seen in the sensors from one vendor, which are operated in a non-inverted mode with parallel clocks that typically operate between 0 and 10 Volts relative to substrate. The other vendor's sensors are operated with inversion of the parallel clocks, using voltages of -8 Volts and +2 Volts. If any charge were to be collected at the $\mathrm{Si}-\mathrm{SiO} 2$ interface it would recombine with holes during the next cocking cycle. LSST is investigating the use of a period of inversion prior to exposure, which might serve to 'erase' the residual image in a non-inverted sensor.

\subsection{Charge diffusion and charge transfer efficiency measurement}

Detectors such as the LSST prototypes present challenges in making some standard characterization measurements. An example is the measurement of charge transfer efficiency (CTE). The standard tool for measurement of CTE in CCDs is the analysis of the peak pixel intensity of ${ }^{55} \mathrm{Fe}$-ray generated charge packets collected in the silicon. We will not describe the process in detail here as it is well documented in the literature ${ }^{2,12,14}$.

For relatively thin devices which present relatively little opportunity for charge to diffuse while moving from the conversion point to the collection point, there is a high probability that all the electrons in a charge packet will be 
captured in a single pixel, allowing for a direct measure of the system gain as well as a determination of CTE by measurement of the peak pixel signal as a function of the position of the pixel in which the charge was collected and therefore the amount of signal that was lost from the pixel as it was transferred to the output node. Note that this technique is sensitive to charge that is lost due to capture in both short and long time constant traps as it excludes all signal not found in the brightest pixel.

Thick devices make the measurement much more difficult. The shallow conversion depth of the ${ }^{55} \mathrm{Fe} x$-rays that are typically used means that the photoelectrons in the charge packet must travel a greater distance from the conversion site to reach the collection site in the buried channel. Diffusion of charge in the silicon during this time makes the detection of 'single pixel' charge packets much less likely. LSST researchers have made a detailed study of the charge diffusion as a function of backside bias voltage. Using ${ }^{55} \mathrm{Fe}$ charge packets as probes, we measure the FWHM of the collected packet images. A measure of the diffusion of charge as a function of backside bias voltage is shown in Figure 4.

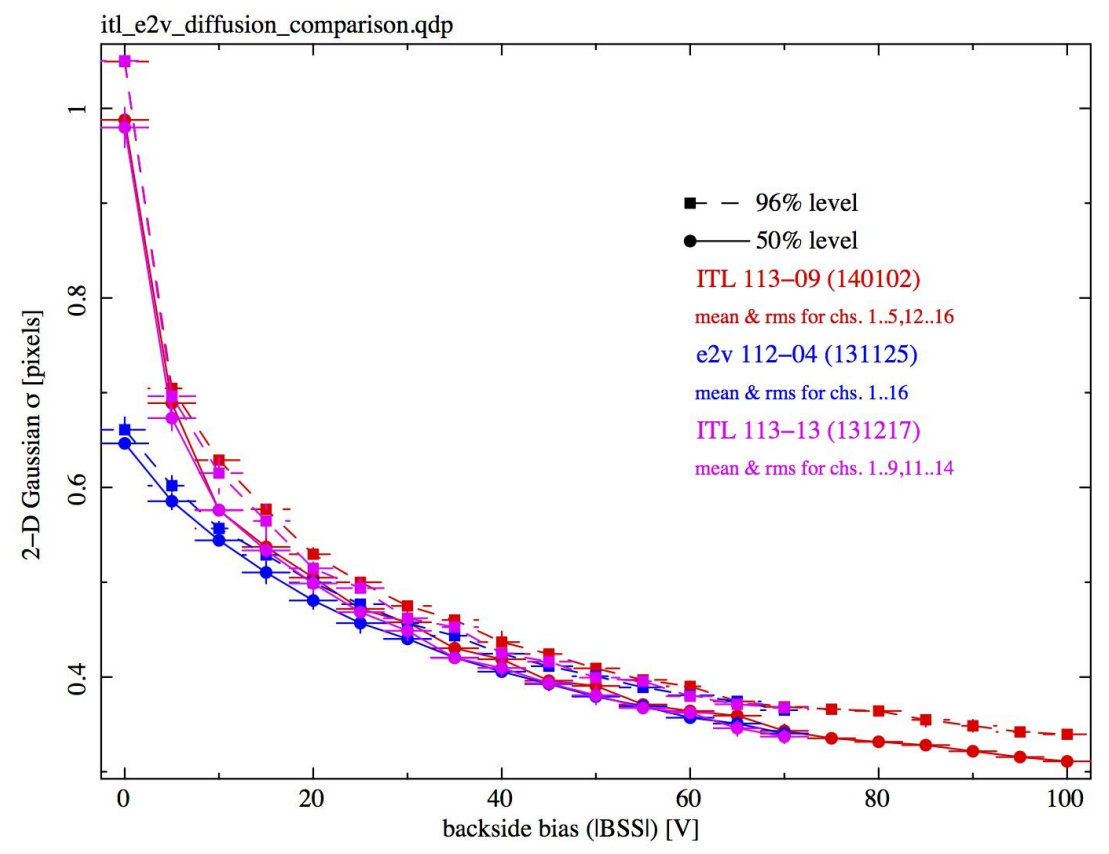

Figure 4: Charge diffusion as a function of backside bias voltage. Results for three LSST prototype sensors are shown. Note that due to differences in the voltages applied to the parallel clocks, the ITL data are shifted roughly $5 \mathrm{~V}$ towards the right side of the plot.

Even when a backside bias voltage of $-70 \mathrm{~V}$ is applied there are few 'single pixel events', making it difficult to use traditional methods of data analysis to measure the CTE of the device. Given that, LSST has decided that the diffusion of charge that occurs in these thick, high-resistivity CCDs makes it impossible to use the standard ${ }^{55} \mathrm{Fe}$ test for CTE measurement. LSST researchers have investigated various methods, including shape analysis of the collected charge packets, to provide a precise measure of CTE in these types of detectors. So far, these investigations have not produced a robust method. Other proposed solutions, including the summation of the charges in adjacent pixels, serve to destroy the information about short time constant traps. LSST will be using the 'extended pixel edge response' method that is well documented in the literature ${ }^{12}$. Work on other methods continues. 


\section{ELECTROSTATIC EFFECTS AND SENSOR PERFORMANCE}

\subsection{Lateral electric fields and charge re-distribution}

The fundamental assumption that is frequently made about the operation of a two dimensional CCD image sensor is that the charge collected in the potential wells, which is then transferred to the output node for digitization, is an accurate representation of the distribution of photons that impinged upon the exposed surface of the device. For this to be the case, the electrons generated by photo-conversion in the silicon must travel in a 'straight line' from the conversion site to the nearest potential well in the buried channel. The electric fields that cause the electron drift must be normal to the surface of the device and sufficiently strong so as to prevent significant spatial diffusion during travel. In front illuminated or backside illuminated devices with a relatively thin substrate, the electric fields generated by the channel stops and the voltages applied to the image array ('parallel') clock lines provide these electric fields. In thicker backside illuminated devices, such as the LSST prototype sensors, an additional voltage is required. The 'backside' surface of the device is manufactured so as to allow a static voltage to be applied. The potential difference between this surface and the potentials at the 'front' of the sensor, where charge is collected, which are set by the potentials applied to the image array clocks and the potential of the channel stops, is what establishes the electric fields that create the pixel boundaries. Any distortion of these electric fields caused by static or dynamic voltage gradients generated either internally within the silicon or externally, can affect the geometry of the electric fields and therefore the effective mapping of the location of the photo-conversion to the location of charge collection. Any such change to the conversion to collection mapping creates a geometric distortion error in the digitized image data. Given that CCD image sensors consist of pixilated arrays of collection regions and that any distortion of the mapping of conversion site to collection site is only significant if the distortion is large enough that charge is collected in a pixel other than that which is geometrically nearest, we refer to the effect as charge redistribution.

If no sources of voltage potentials existed other than the 'backside' bias, that of the channel stops, and the potentials applied to the image array clocks, it would be expected that the mapping of photo conversion to charge collection would be accomplished with no charge redistribution. However, there are other sources of potential that can affect the geometry of the electric fields. These sources can include static 'structural' features that are part of the device design and dynamic sources related to the charge collection process itself. Recent work by a number of researchers ${ }^{4,5,7,9,10,15,16}$ has exposed more clearly the effects that both static and dynamic electric fields can have on the effective mapping of photoconversion location to charge collection location.

As example of structural design features that can produce charge redistribution, we present the electrostatic effects of the 'scupper' feature that is frequently implemented to guard against charge generated at the periphery of the device from contaminating the signal in the imaging array.

A subtler static structural feature is the variation in doping concentrations in the silicon substrate. This variation leads to a deformation of the electric fields that guide the electrons generated by photo-conversion to the collection site in the potential wells. This leads to a slight variation in the 'effective' pixel sizes: a variation in the volume of silicon in the conversion region that will lead to an electron being collected in a particular pixel's potential well.

Dynamic potentials can also create electric field disturbance significant enough to produce geometric distortion with the potential to affect LSST science goals. In particular, we refer to the charge redistribution caused by electric fields generated by integrated photocurrent that can cause subsequent distortions in the conversion to collection mapping. This effect, recently given the name of the 'Brighter-Fatter' effect, relates the manner in which charge that has been collected in the potential wells of the CCD sensor can affect the electric fields, and therefore the charge redistribution, of the sensor subsequently.

\subsection{Lateral field distortion at the edges of the detector}

As do many other CCDs, the LSST prototype image sensors incorporate a charge drain that encircles the imaging area in order to collect any charge that is generated outside of the pixel array due to either dark current or photo-conversion. This 'Guard Drain' or 'Scupper', as such structures are known, is held at a high positive voltage potential, between 20 and 30 volts for LSST prototype sensors. This voltage produces an electric field lateral to the electron drift direction. Close to the edges of the device this electric field causes a distortion in the fields that are created by the channel stops, parallel clocks, and backside bias. The effects of charge redistribution can be seen in images acquired with a flat field illumination. A section of one such image is shown in Figure 5 taken from one corner of an image segment at the edge of 
the CCD. The scupper is located to the right of and below the pixels in the image section shown. The affect of the scupper can be seen as it reduces the apparent photometric sensitivity of the device as shown in Figure 6.

The charge redistribution in this case is so significant as to cause as much as a $65 \%$ reduction in the apparent sensitivity of the imaging column at the edge of the array. LSST has established a specification for defective columns that defines any column that contains more than 100 contiguous pixels with measured sensitivity below $80 \%$ of the detector mean sensitivity to be a 'dark column' defect. The charge redistribution caused by the scupper causes three or four columns on either edge of the detector to meet this definition as a defect. Similarly, there are rows near the serial registers on those edges of the imaging array that also contain pixels with sensitivity below $80 \%$ of the mean. LSST has defined no 'dark row' specification, so the pixels in these rows all fall under the 'dark pixel' specification.

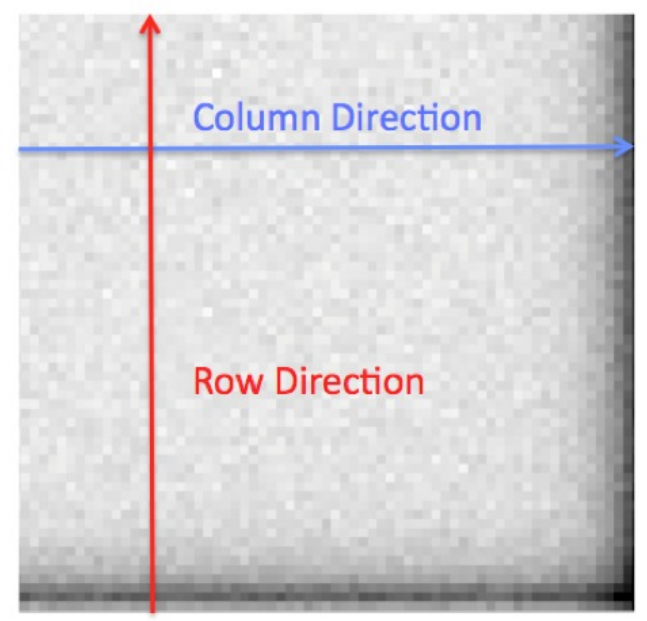

Figure 5: Flat field variations at the edge of the imaging array of an LSST prototype CCD. The 'scupper' or 'Guard Drain' structure is located to the bottom and right of the image array pixels shown in this figure.

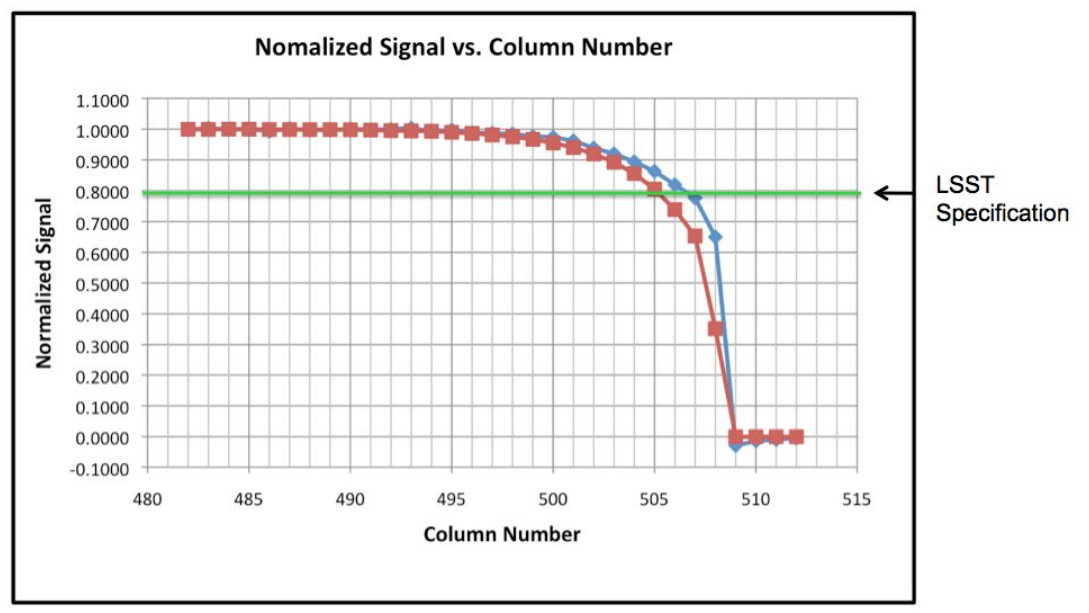

Figure 6: Photometric Response near the Vertical Edges of the CCD. The results for two sensors of different design and manufacture are shown. The data have been aligned such that the last imaging column is number 508 .

That this effect is caused by the 'scupper' (or 'Guard Drain') voltage can be shown by varying the applied voltage and measuring the magnitude of the reduction in apparent sensitivity. For this test identical flat field images were acquired 
with different applied scupper voltages. The result of this experiment is shown in Figure 7. There is a nearly linear relationship between the magnitude of the photometric response reduction and the applied voltage.

Assuming that the effect is caused by charge generated by photo-conversion being diverted as it travels from the photoconversion site to the collection site, it would be reasonable to expect that the magnitude of the effect would have a dependence on the wavelength of the photon flux. As longer wavelength photons convert deeper in the silicon (and therefore closer to the collection site) they should have less drift time before collection. In order to study this, we produced a number of flat field images at various wavelengths with a pass-band width of $\sim 10 \mathrm{~nm}$. Figure 8 shows the normalized results. It can be seen that the edge effect occurs at all the wavelengths and that it is somewhat less pronounced at longer wavelengths.

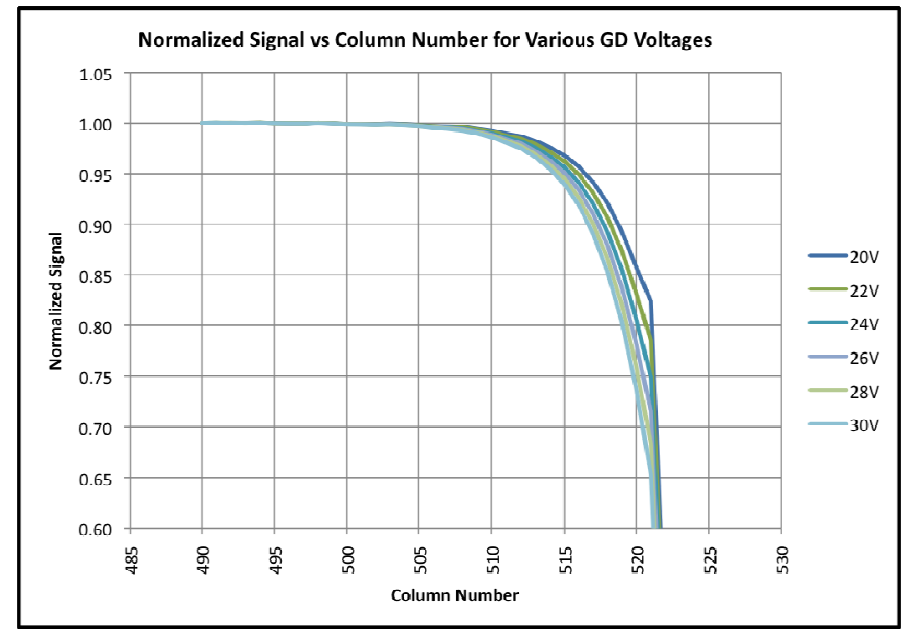

Figure 7: Photometric effects at the edge of the sensor image array. Lateral electric fields caused by the Guard Drain voltage create a distortion in the effective pixel sizes near the edge of the array.

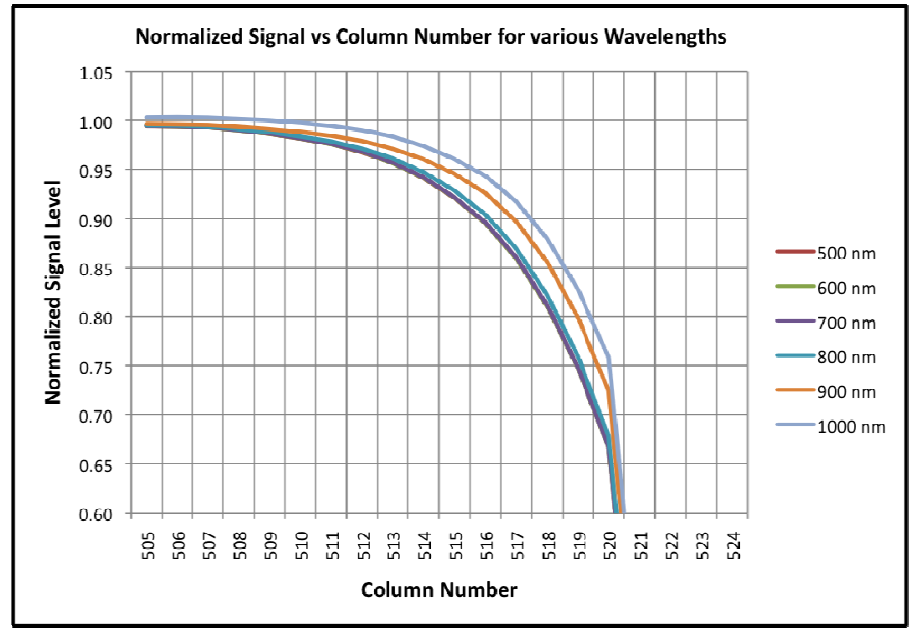

Figure 8: Signal versus wavelength at the edge of the imaging array. There is a wavelength dependence on the photometric distortion caused by the Guard Drain voltage.

Charge redistribution, as opposed to a reduction in apparent photometric sensitivity, should also have a positional or astrometric effect. In order to study this, images of a small focused spot were acquired at various positions at differing distances from the edge of the device. The row and column centroids of the detected spots were then calculated using SExtractor, a standard tool in the astronomical community. A linear fit was then made to those centroids and errors computed for each measured spot location.

As can be seen in Figure 7, the column component (x direction) of the measured centroid deviates increasingly from the linear fit as the spot moves towards column 520, the edge of the device after which the spot image is clipped and an 
accurate centroid cannot be calculated. That the astrometric error is induced by charge redistribution can also be inferred from the fact that the shape of the spot is also distorted as it approaches the edge of the device.
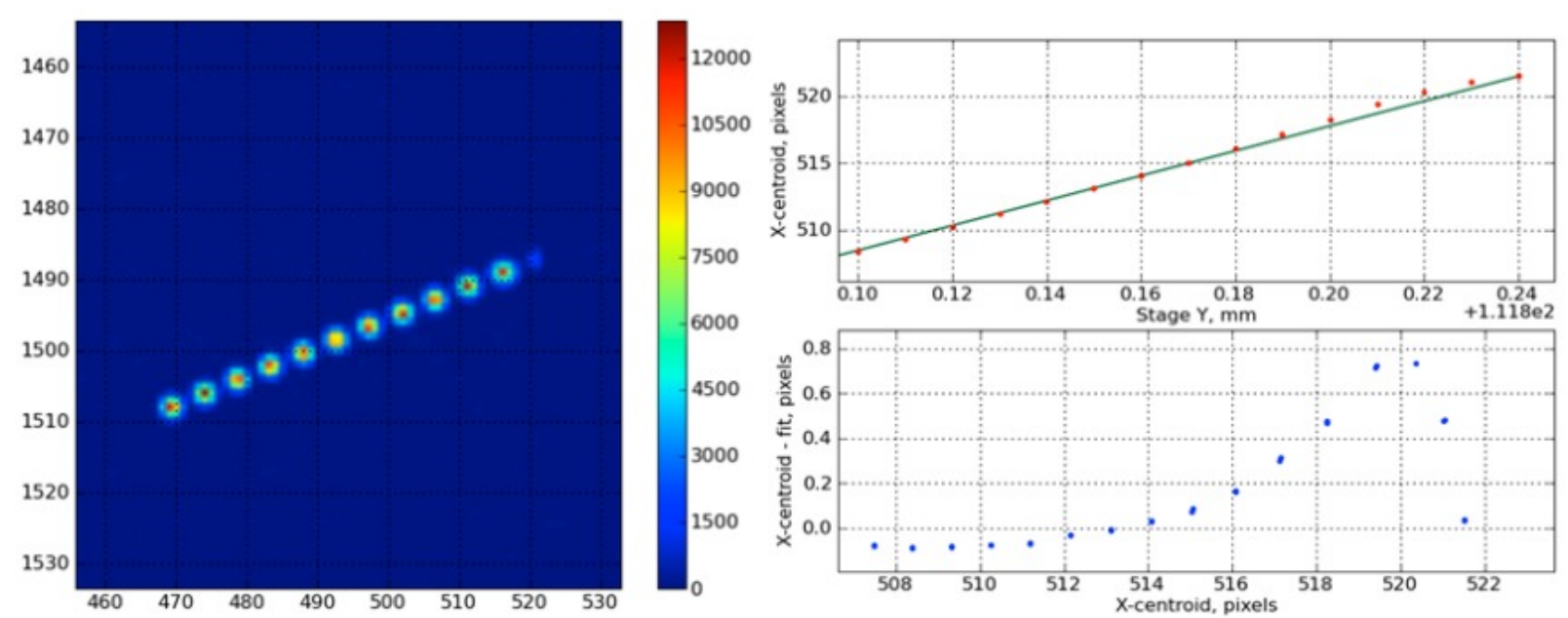

Figure 9. Spot images and detected astrometric errors at CCD edge caused by electron interaction with fields caused by the 'Guard Drain' voltage that surrounds the imaging area of the device. The image on the left of the figure shows the superposition of spot images. The $\mathrm{Y}$-axis is the row number and the $\mathrm{X}$-axis is the column number. The last imaging column is number 522 .

\subsection{Substrate resistivity variation}

Another static source of potential that can change the effective size of CCD pixels by modifying the electric fields that define the pixel boundaries is variation in the density of dopant and impurities in the silicon substrate. These variations typically have a circular symmetry about the center of the silicon wafer upon which the CCD is manufactured.

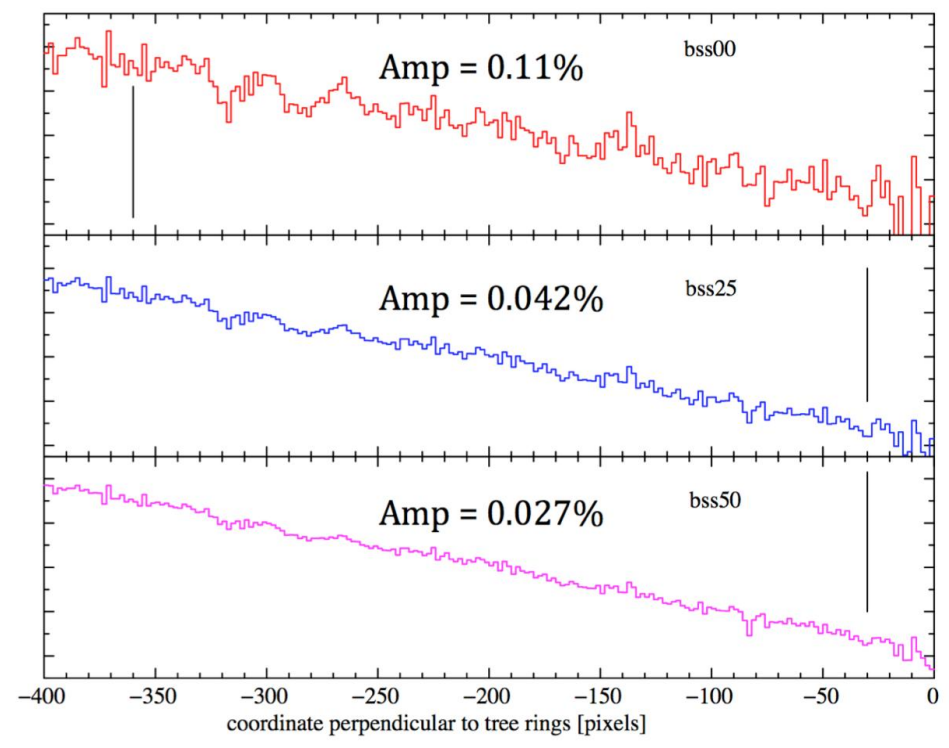

Figure 10: Amplitude of photometric variation caused by the distortion caused by variation in effective pixel size caused by variation in resistivity of the silicon wafer on which the CCD is manufactured. Results for three different values of the backside bias voltage are shown. For reference, the black bars represent a $1 \%$ variation in pixel response. The small overall slope in all three cases is caused by non-uniform illumination. 
That the effect is caused by effective pixel size and not quantum efficiency variations can be seen by measuring the photometric sensitivity variations as a function of the electric field strength. To do so we plot the measured photometric sensitivity of the detector in the radial direction, orthogonal to the 'tree ring' structure. By varying the backside bias voltage we also vary the strength of the electric fields that define the pixel boundaries. As can be seen in Figure 10, the amplitude of the sensitivity variations is a strong function of the backside bias voltage. As the voltage difference between the back surface of the CCD and the collection site is increased, the sensitivity variations are diminished. Results for three different values of the backside bias voltage are shown. For reference, the black bars represent a $1 \%$ variation in pixel response. The small overall slope in all three cases is caused by non-uniform illumination

\subsection{Accumulated Charge Generated Electric Field Effects (Bright-Fatter)}

In order to accurately make the measurements that LSST science goals require, it is important to fully understand the point spread function (PSF) of the system. This is critically important for making 'weak lensing' measurements: the measurement of shear of background galaxy images caused by mass distributions along the line of sight. Computational systems for post processing of image data typically assume that the PSF of the system can be determined accurately by analysis of bright stellar images. Errors in the determination of the PSF based on stellar object shape analysis will lead to systematic errors in the shear measurement. Typically, precise measure of shear requires an understanding of the system PSF to the $0.1 \%$ level. In some cases an additional factor of 10 in precision may be required. Recent work at LSST laboratories has shown that charge redistribution caused by electric field disturbance due to the presence of previously collected charge that is present in the potential wells of the device can have an effect on the effective size of the CCD pixels and thus the determination of PSF at the $1 \%$ level. In the case of spot image studies, this has been referred to as the 'brighter-fatter' effect ${ }^{11}$.

In order to study the effects of charge redistribution on PSF measurement, an experimental setup was constructed that allowed the imaging of circular sources. A precision single pinhole mask was illuminated from the rear with a stable, narrow wavelength pass-band, diffuse and uniform light source created with a lamp, monochromator, and integrating sphere. The illuminated pinhole was then imaged onto the surface of the CCD using a simple relay lens. Images were acquired at various wavelengths and with varying intensities. Images with peak spot intensities ranging from just a few electrons per pixel to saturation were acquired. Multiple images at each intensity were acquired to allow for stacking of frames to increase signal to noise ratio.

Figure 11 shows one result of this test. The image on the left is a 200 second exposure, the center image is the average of twenty 20 second exposures and the image on the right is the difference after scaling and re-centering. A logarithmic scaling is used to emphasize the low intensity portions of the spot image. The fact that the central pixels of the brighter spots are reduced in amplitude is obvious as is the fact that the surrounding pixels in the brighter image contain an excess of charge. This is the 'brighter-fatter' effect.
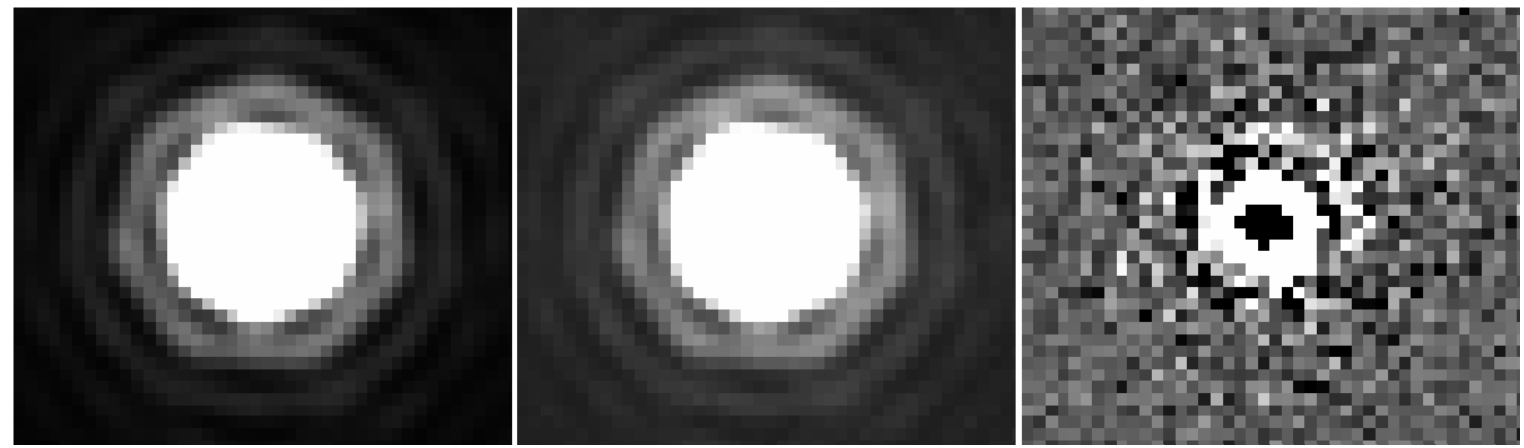

Figure 11: Brighter-Fatter effect revealed by difference imaging. 
Analysis of spot size as a function of intensity reveals a linear relationship. Figure 12 shows the size of a number of spot images as function of the spot intensity for an E2V CCD250 device. This effect has been seen in earlier LSST prototype sensors as well. Figure 13 shows a similar result for an STA1920A device.
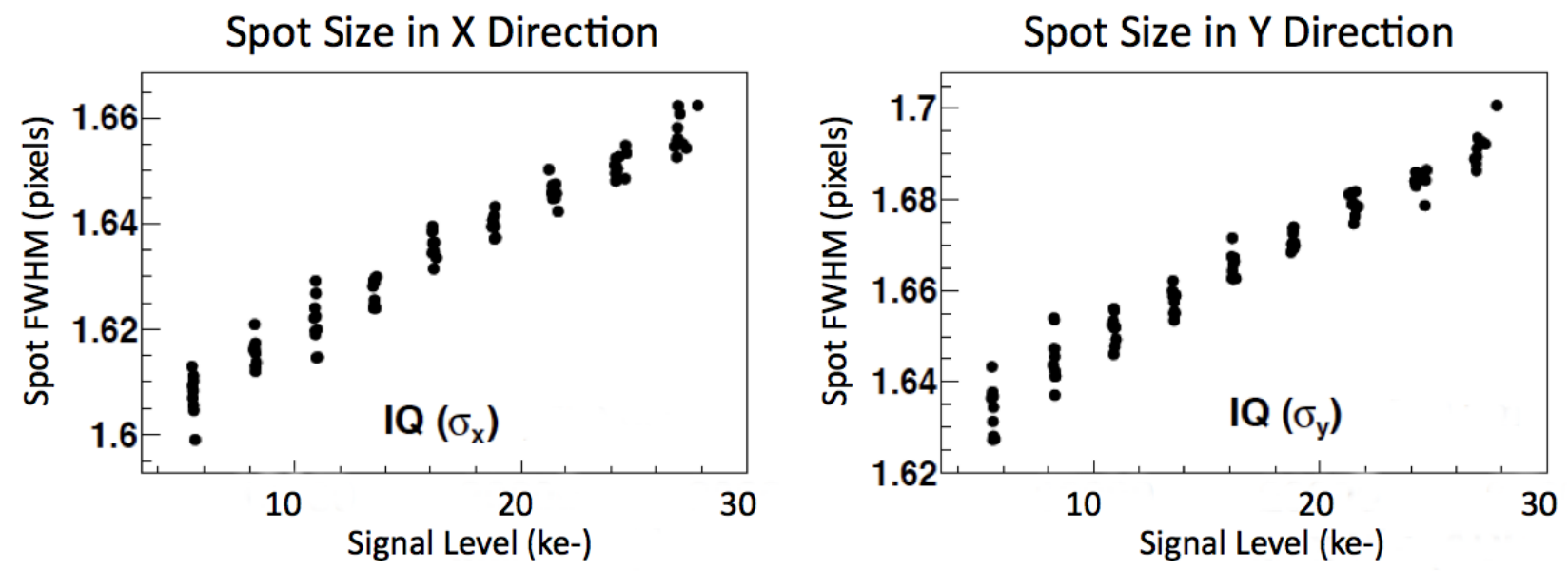

Figure 12: Measured spot image size vs. intensity for an E2V250 CCD. The measured full width half maximum spot size is plotted against the peak signal level in the spot image. The dependence of the spot diameter as a function of intensity is seen to be a linear function.

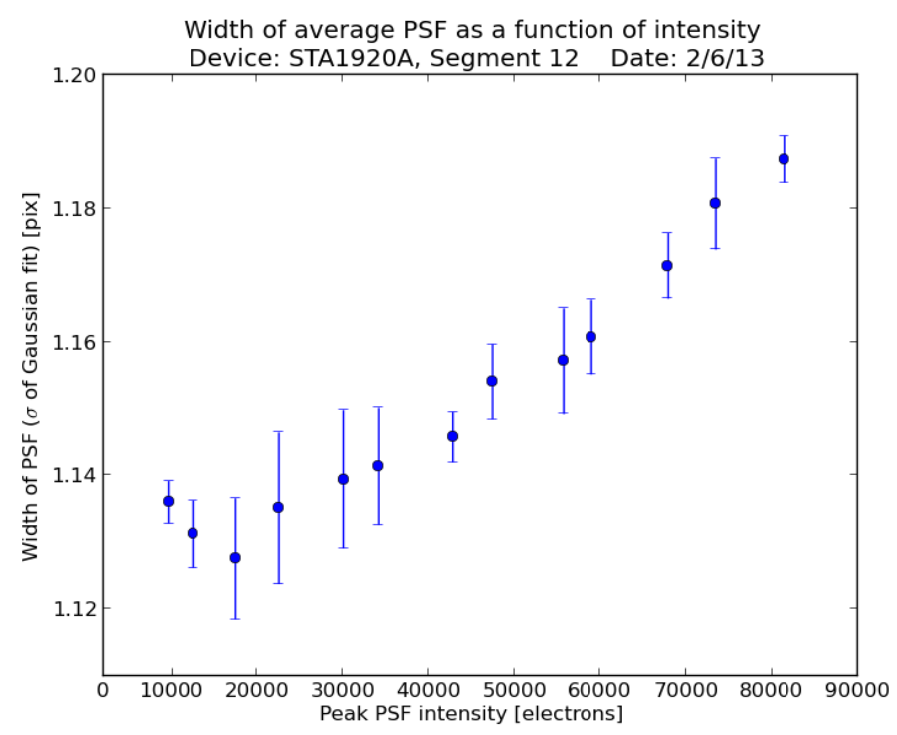

Figure 13: Measured spot image size vs. intensity for an STA1920 CCD using a similar laboratory test setup.

The 'brighter-fatter' effect appears to be caused by charge collected into a pixel modifying the electric fields in that section of the silicon. LSST researchers at IN2P3 have developed a simple model for the field lines in a CCD sensor near the buried channel. Figure 14 shows, on the left, the symmetric electric field lines that one would expect in a biased but 'empty' CCD. Only the 20 micrometers of vertical depth closest to the buried channel collection sites are shown in the diagram. LSST Prototype CCDs are 100 micrometers thick. Pixel boundaries are at 5, 15, and 25 um respectively. On the right half of the figure we see the field lines as distorted by a charge packet of $50 \mathrm{ke}$ - stored in the rightmost pixel. As can be seen in the diagram, the field lines have been distorted sufficiently for some charge that would have been captured in the rightmost pixel would now be captured in the pixel to its left. 
The electrostatic model has been used to simulate the effective pixel size that might be expected for different quantities of electric charge and therefore the spot image size that could be expected. Figure 15 shows a comparison of the measurements to the prediction from the model.
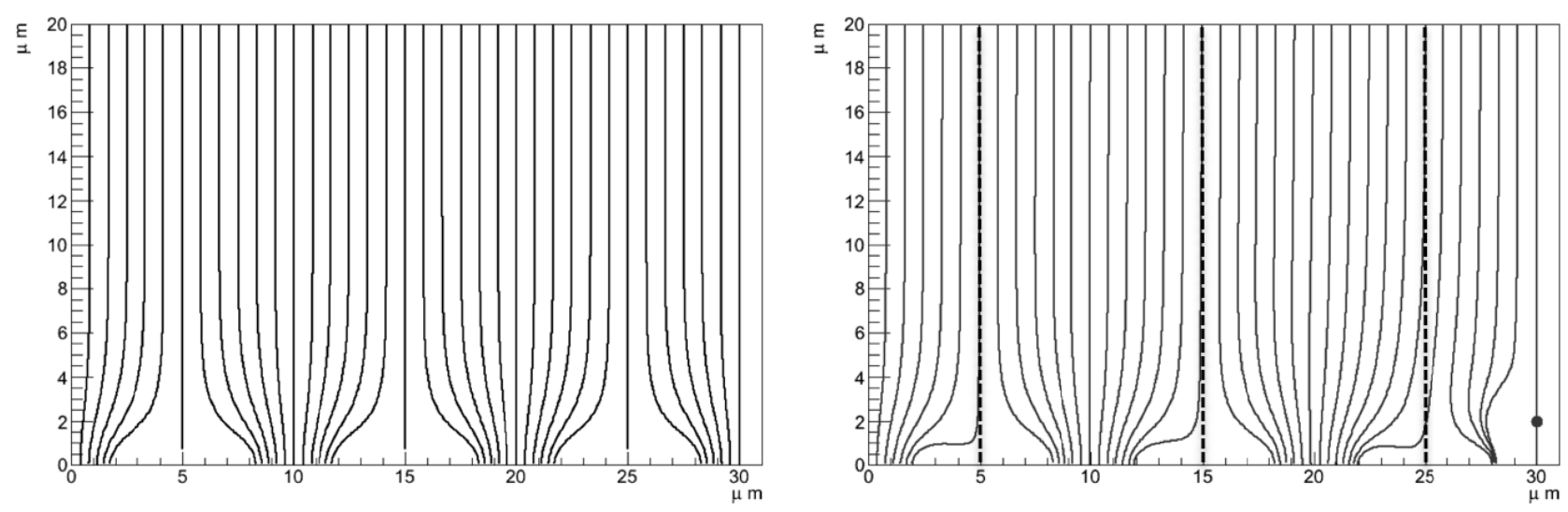

Figure 14: Electric fields without and with collected charge present. The figures show the electric fields in the 20 micrometers closest to the collection sites at the surface of the CCD. The LSST prototype sensors are 100 micrometers thick.

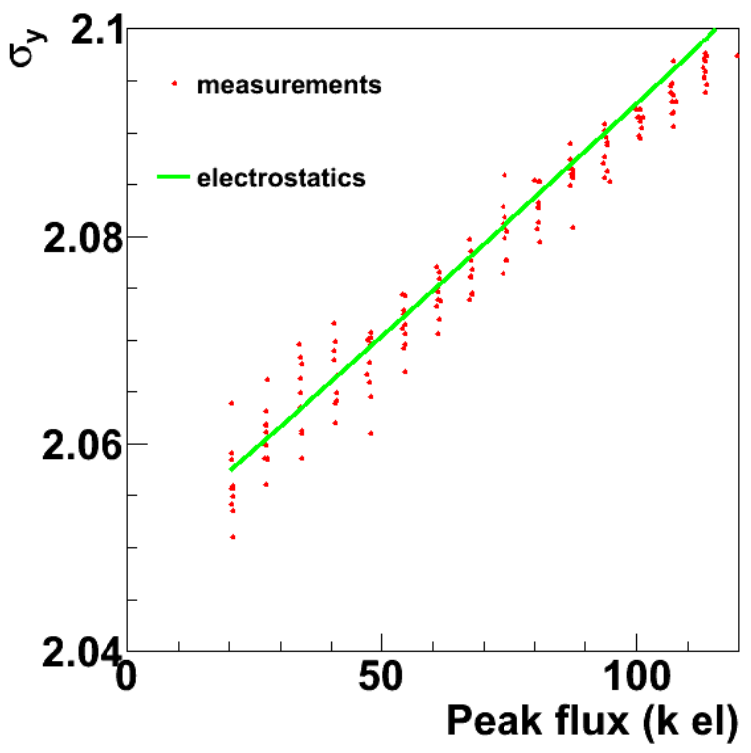

Figure 15: Comparison of electrostatic model to laboratory measurements

\subsection{Correlated charge and the photon transfer curve}

The 'photon transfer curve', in which the variance of pixel values is plotted as a function of mean signal level, has been a tool of the trade for CCD testing for many years ${ }^{[2]}$. It is outside the scope of this paper to present a detailed description of the technique or its uses. It has previously been presented that for thick, high resistivity CCDs there is a non-linear effect in which the variance does not track the mean in apparent violation of Poisson statistics ${ }^{7,9}$. Figure 16 shows a photon transfer plot for one amplifier of an LSST prototype CCD. 


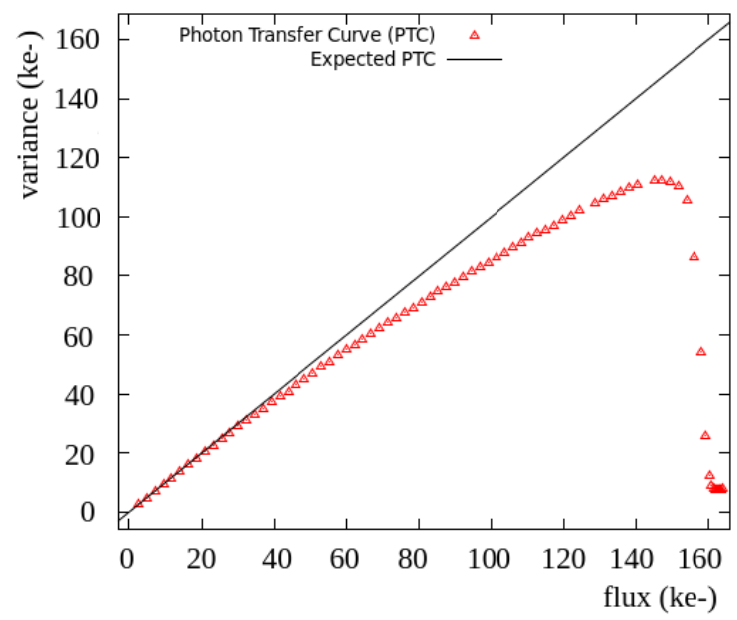

Figure 16: Photon transfer curve for an LSST prototype CCD amplifier

LSST scientists at LPNHE in Paris have posited ${ }^{11,4}$ that the cause of the non-linearity in the photon transfer curve is the same as for the 'brighter-fatter' effect seen for spot images, described above. If the charge in one pixel has an affect on the charge collection properties of adjacent pixels, causing a change in the effective pixel size, there will be a correlation in the signal levels and therefore a reduction in the variance. This apparent violation of Poisson statistics is greatly reduced when the data are binned together into 'super-pixels' during calculations. This would seem to indicate that there are statistical correlations between pixels in flat field images.

We have developed software for the measurement of this correlation and applied it to LSST flat field images. Figure 17 shows the correlation coefficients for adjacent pixels in flat field images with different parallel clock voltages applied. As the parallel clock voltages help to define the pixel boundaries, one would expect that higher voltages applied to the parallel clock lines would result in lower correlation. This is what is observed.

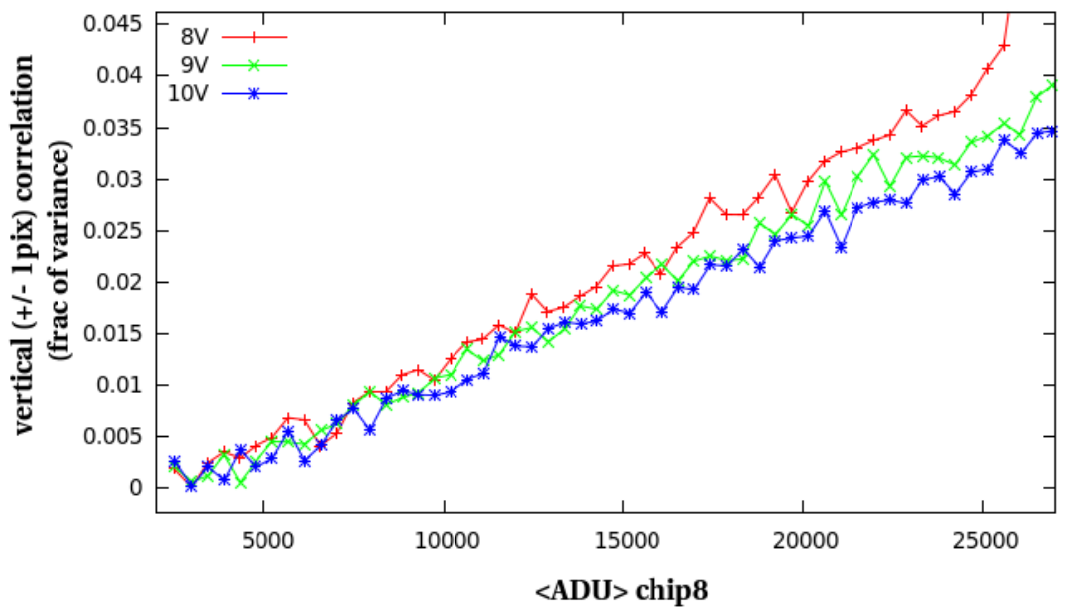

Figure 17: Vertical Correlation as a function of signal level for various parallel clock voltages

Given a measure of the correlation coefficients, it is possible to calculate the inverse process and recover the linearity of the photon transfer curve. The result of this calculation is shown in Figure 18. The upper plot shows the original calculated variance that does not include the covariance terms and the results when the covariance of adjacent pixels is 
included, and when the covariance terms for pixels up to three rows or columns away are included. The lower plot shows the residuals from linear fits to the data in the upper plot. Note that even the unexplained dip towards the top end of the original curve is corrected.
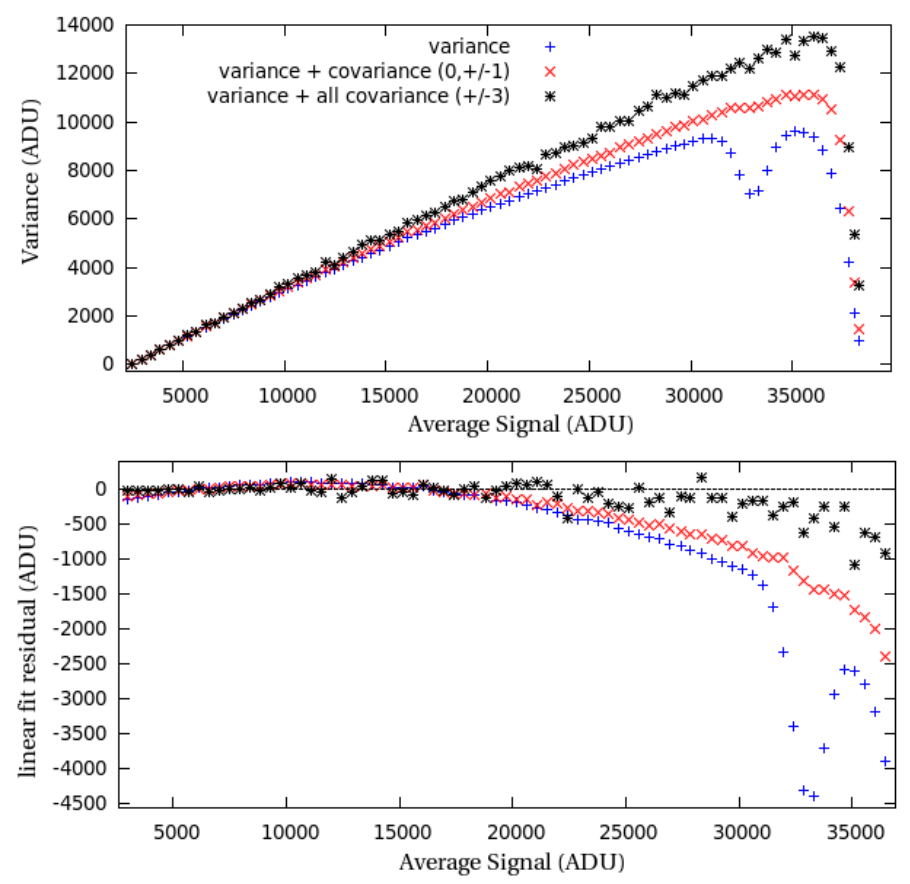

Figure 18: Photon transfer curves corrected for pixel to pixel correlation

\section{SENSOR PERFORMANCE IMPACT ON LSST SCIENCE}

There are a variety of ways in which charge redistribution caused by lateral electric fields in a silicon CCD image sensor can affect the photometric and astrometric performance of a sensor. The use of thicker, high resistivity silicon substrates for the newest generations of image sensors has brought these possibilities to the forefront. In retrospect, these effects were always present at some level. Recent analysis of data from existing camera systems has shown that residual errors caused by the changes in effective pixel size, both static and dynamic, place a limit on system performance if no method is in place to account for them ${ }^{4}$. As stated in the introduction, many of the scientific goals of the LSST project require extreme precision in either phototmetric or astrometric measurement or both. Some key science missions of the project serve as examples.

Measurement of the history of cosmic expansion due to dark energy is done using several types of probes, each of which is sensitive to systematic errors in photometry and/or astrometry. The use of type Ia supernovae as 'standard candles' requires relative photometry with better than $1 \%$ precision. Currently, systematic errors, some of them produced in the data reduction process, limit the precision with which these measurements can be made. For example, the LSST team has developed a simulation of the charge displacement effects observed at the edges of the detector that closely matches the observed phenomenon. A simulated image containing a field of circular objects of identical flux level and similar in size and geometry to the images that LSST will produce for stellar sources was created and then 'warped' by modification of the effective pixel size and shape as seen in the laboratory flat fields. Then, standard flat fielding photometric correction was applied. Figure 19 shows the results of this exercise. In the upper plot, we see the measured magnitude for the simulated sources as they approach the edge of the detector prior to any attempt at flux calibration via a 'flat field' gain correction. The effects of the electric fields caused by the scupper voltage can clearly be seen. The lower plot shows the measured source magnitudes after a simple flat fielding correction is applied. The correction process would have a similar distorting effect anywhere that effective pixel sizes are affected by lateral electric fields. 

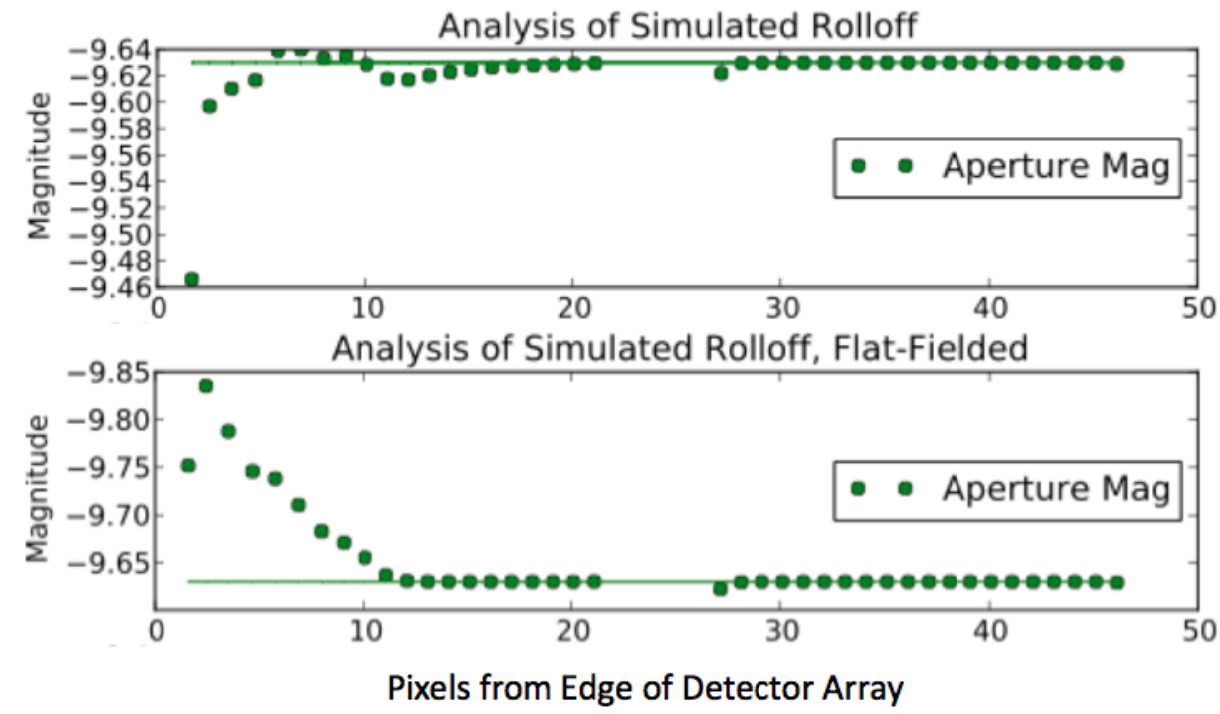

Figure 19: Effect of flat fielding on aperture photometry of simulated stellar sources that are affected by lateral electric fields at the edge of a detector caused by the scupper voltage

In addition to causing an error in the calculated stellar magnitude of the objects, the flat field gain correction also creates an error in the measured position of the spot images. Figure 20 shows the position error as a function of the distance of the center of the spot image from the edge of the device. For simulated stellar images, the flat field gain correction process is shown in Figures 19 and 20 to have created errors in both magnitude and position.

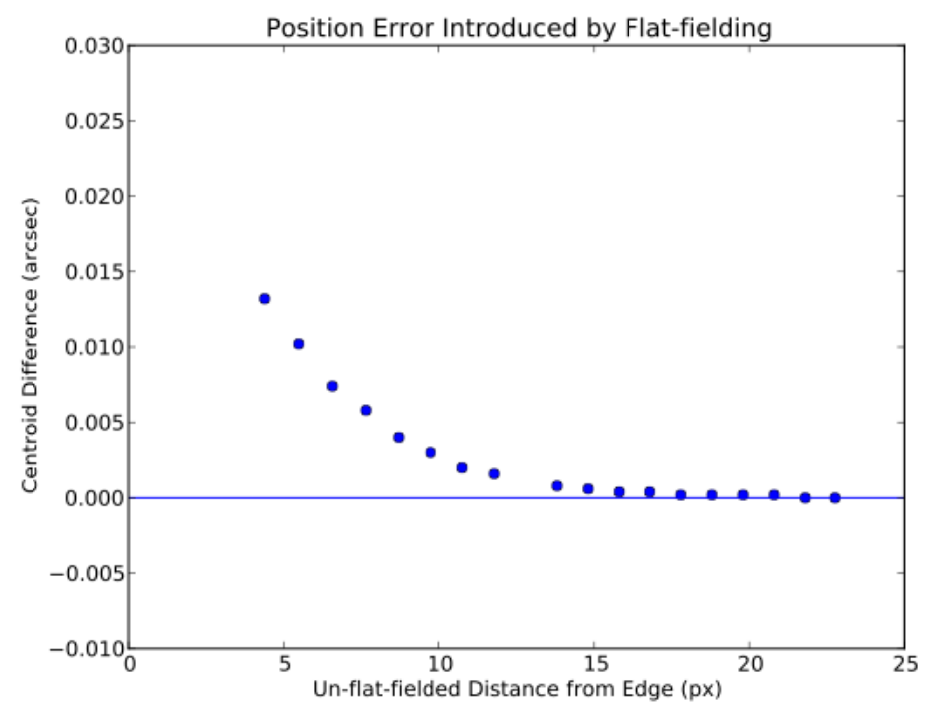

Figure 20: Position error created by applying flat field correction to simulated sources near the edge of a detector

A key probe of dark energy is the mapping of dark matter concentrations via measurement of cosmic shear and convergence in the images of background galaxies caused by weak gravitational lensing of those sources due to mass concentrations along the line of sight. This requires high precision measurements of the ellipticity of those background galaxies. These measurements require a detailed understanding of the point spread function of the system. Any variation 
in effective pixel size of the sensor as a function of pixel position, such as those caused by the types of lateral field effects discussed in this paper, can cause spatial variation of the PSF across the imaged field. This will have to be measured and taken into account for proper galaxy shape determination. In addition, the intensity dependence of the PSF of point sources, the 'bright-fatter' effect discussed above, makes it difficult to use bright stars, with their well defined PSF, to calibrate the shape parameters of the much fainter galaxy sources that are of interest in the shear measurements. LSST will depend upon the joint analysis of many images of each source (as many as 800 or more) in order to increase the signal to noise ratio. The varying PSF as the source is imaged via different parts of the detectors must be modeled via measurements of stars and a 3-D model of charge transport.

The precision that LSST science requires in both photometric and astrometric measurement of astronomical sources will require an understanding of the performance of the CCD sensors that meets or exceeds that of prior experiments. Ongoing study of the characteristics of the sensors will be required in order to properly reduce the acquired image data. Such data reduction must take into account the variety of sources of image distortion that affect the mapping of the optical source locations in the focal plane to the measured locations after the converted photo-electrons have traversed the electric fields in the silicon on their way to the potential wells that define the pixels in the digitized image data.

\section{ACKNOWLEDGMENTS}

LSST project activities are supported in part by the National Science Foundation through Cooperative Support Agreement (CSA) Award No. AST-1227061 under Governing Cooperative Agreement 1258333 managed by the Association of Universities for Research in Astronomy (AURA), and the Department of Energy under contract with the SLAC National Accelerator Laboratory. Additional LSST funding comes from private donations, grants to universities, and in-kind support from LSSTC Institutional Members.

\section{REFERENCES}

[1] LSST Science Collaborations and LSST Project, [LSST Science Book Version 2.0], arXiv:0912.0201 (2009).

[2] J.R. Janesick, [Scientific Charge-Couple Devices,] SPIE Press, Bellingham, WA (2001).

[3] P. Kubánek et al., "Use of RTS2 for LSST multiple channel CCD characterization”, Proc. SPIE 8451 (2012).

[4] A. Guyonnet, P. Astier, P. Antilogus, N. Regnault and P. Doherty, 2014 "Evidence for self interaction of charge distribution in CCDs", Manuscript in preparation

[5] I. Kotov et al., "Study of pixel area variations in fully depleted thick CCD”, Proc. SPIE 7742 , 774206-1 (2010).

[6] R. Smith \& G. Rahmer, "Pixel area variation in CCDs and implications for precision photometry", Proc. SPIE 7021, 70212A-1, (2008).

[7] M. Downing et al., "Bulk silicon CCDs, point spread function and photon transfer curves: CCD testing activities at ESO”, Proc. SPIE. 76 (2005)

[8] P. O'Connor, "Spot Scan Probe of Lateral Field Effects in a Thick Fully-Depleted CCD”, JINST 9 C03033 in proceeding for Precision Astronomy with Fully Depleted CCDs, 18-19 November 2013, Brookhaven National Laboratory, U.S.A. (2014).

[9] M. Downing, B. Baade, P. Sinclaire, S. Deiries, and F. Christen, "CCD riddle: a) signal vs time: linear; b) signal vs variance: non-linear", Proc SPIE 6276-09 (2006).

[10] I.V. Kotov, A.I. Kotov, J. Frank, P. Kubanek, M. Prouza, P. O’Connor et al., "Study of pixel area variations in fully depleted thick CCD”, High Energy, Optical, and Infrared Detectors for Astronomy IV, SPIE 7742 (2010). 
[11] P. Astier, "The brighter-fatter effect and pixel correlations in CCD sensors", JINST 9 C03048 in proceeding for Precision Astronomy with Fully Depleted CCDs, 18-19 November 2013, Brookhaven National Laboratory, U.S.A. (2014).

[12] Waczynski, E. J. Polidan, P. W. Marshall, R. A. Reed, S. D. Johnson, R. J. Hill, G. S. Delo, E. J. Wassell and E. S. Cheng, "A comparison of charge transfer efficiency measurement techniques on proton damaged CCDs for the Hubble Space Telescope Wide-Field Camera 3", IEEE Trans. on Nucl. Sci., vol. 48, no. 6, pp 1807-1814, (2001).

[13] C. Stubbs, "Precision Astronomy with Imperfect Fully Depleted CCDs - An Introduction and a Suggested Lexicon", JINST 9 C03032 in proceeding for Precision Astronomy with Fully Depleted CCDs, 18-19 November 2013, Brookhaven National Laboratory, U.S.A. (2014).

[14] J. Holtzmam et al., "The performance and calibration of WFPC2 on the Hubble Space Telescope", Publ. Astron. Soc. Pac., vol. 107, pp.156-178, (1995).

[15] S.E. Holland, D.E. Groom, N.P. Palaio, R.J. Stover and M. Wei, "Fully depleted, back-illuminated charge-coupled devices fabricated on high-resistivity silicon", IEEE Trans. Elec. Dev. 50225 (2003).

[16]D.E. Groom et al. "Point-spread function in depleted and partially depleted CCDs", Proceedings of The 4th ESO Workshop on Optical Detectors for Astronomy, Garching, Germany, 13-16 September 1999 (1999).

[17] A. Karcher et al., "Measurement of lateral charge diffusion in thick, fully depleted, back-illuminated CCDs", IEEE Trans. Nucl. Sci. 512231 (2004). 